\title{
Orientações para o aleitamento materno e alimentação complementar à criança atendida na puericultura
}

\author{
Breastfeeding and complementary feeding guidance for childcare assistance \\ Orientaciones para la lactancia materna y alimentación complementaria del niño atendido \\ en la puericultura
}

Dayane Pereira da Silva ${ }^{1 *}$, Marina Valente Mascarenhas ${ }^{1}$, Isadora Helena Araújo Silva1 ${ }^{1}$, Lidiane do Nascimento Rodrigues ${ }^{1}$, Vanusa Maria Gomes Napoleão Silva ${ }^{1}$, Edna Maria Camelo Chaves ${ }^{1}$.

\section{RESUMO}

Objetivo: Relatar a vivência das atividades de orientações para o materno e alimentação complementar à criança atendida na puericultura. Relato de experiência: Trata-se de um relato de experiência sobre as atividades do projeto de extensão desenvolvido em uma Unidade Básica de Saúde (UBS), da cidade de Fortaleza-Ceará. O projeto iniciou-se em abril de 2019, com a participação de discentes e docentes do Curso de Enfermagem da universidade. Foram realizadas oficinas divididas em temáticas abordadas na puericultura para com as gestantes e puérperas. Durante a experiência, os participantes realizaram atividades com as mães, possibilitando a atividade de educação em saúde e interação do grupo com as mães. Considerações finais: Foi possível perceber a importância de realizar as ações de extensão, pois possibilitou aos participantes, o aprimoramento de técnicas de educação em saúde essenciais à prática do aleitamento materno e alimentação complementar. Ademais, nota-se que o conhecimento obtido com as ações do projeto possui relevância para a formação dos futuros profissionais.

Palavras-chave: Puericultura, Enfermagem, Atenção básica, Aleitamento materno, Alimentação complementar.

\section{ABSTRACT}

Objective: Report the experience of activities of guidance for the mother and complementary feeding to the child assisted in childcare. Experience report: This is an experience report on the activities of the extension project developed in a Basic Health Unit (UBS) in the city of Fortaleza-Ceará. The project began in April 2019, with the participation of students and professors from the university's Nursing Course. Workshops were held divided into themes addressed in childcare for pregnant and postpartum women. During the experience, the participants carried out activities with the mothers, enabling the activity of health education and group interaction with the mothers. Final considerations: It was possible to realize the importance of carrying out the extension actions, as it enabled the participants to improve health education techniques essential to the practice of breastfeeding and complementary feeding. Furthermore, it is noted that the knowledge obtained from the project's actions is relevant for the training of future professionals.

Key words: Childcare, Nursing, Basic attention, Breast feeding, Complementary food.

\section{RESUMEN}

Objetivo: Informar la experiencia de las actividades de orientación a la madre y alimentación complementaria al niño asistido en el cuidado infantil. Informe de experiencia: Este es un informe de experiencia sobre las

\footnotetext{
1 Universidade Estadual do Ceará, Fortaleza - CE. *E-mail: dayane.pereira@aluno.uece.br
} 
actividades del proyecto de extensión desarrollado en una Unidad Básica de Salud (UBS) en la ciudad de Fortaleza-Ceará. El proyecto se inició en abril de 2019, con la participación de estudiantes y profesores del Curso de Enfermería de la universidad. Se realizaron talleres divididos en temas abordados en el cuidado infantil para mujeres embarazadas y posparto. Durante la experiencia, los participantes realizaron actividades con las madres, posibilitando la actividad de educación para la salud y la interacción grupal con las madres. Consideraciones finales: Se pudo reconocer la importancia de realizar las acciones de extensión, ya que permitió a los participantes mejorar las técnicas de educación en salud esenciales para la práctica de la lactancia materna y la alimentación complementaria. Además, se destaca que el conocimiento obtenido de las acciones del proyecto es relevante para la formación de los futuros profesionales.

Palabras clave: Cuidado de niños, Enfermería, Atención primaria, Lactancia materna, Alimentación complementaria.

\section{INTRODUÇÃO}

A puericultura é um programa na área da saúde da criança, no âmbito da Atenção Básica que tem por objetivo acompanhar o crescimento e o desenvolvimento infantil; observar a cobertura vacinal; estimular a prática do aleitamento materno exclusivo até o sexto mês de vida; avaliar desenvolvimento neuropsicomotor e função auditiva; prevenir doenças prevalentes em crianças no primeiro ano de vida e orientar a introdução da alimentação complementar (GUBERT FA, et al., 2015).

Esse programa tem por meta atender crianças de diferentes faixas etárias, sendo as de maior prevalência, as menores de um ano. Os perfis delas na Unidade Básica são de maioria de crianças eutróficas, com aleitamento materno realizado por, no máximo, sete meses. Além disso, devem ser acompanhados no momento da consulta por um cuidador, na qual grande parte das vezes quem assume esse papel é a mãe (GAUTERIO DO, et al., 2012).

Assim sendo, para que a criança tenha um acompanhamento adequado de seu crescimento e desenvolvimento satisfatório, é necessário além do cuidado do profissional, o cuidado materno, que deve incluir ações biopsicosocio-ambiental para propiciar à criança condições para que se desenvolva adequadamente (MINISTÉRIO DA SAÚDE, 2018). É importante compreender que, além de sentimentos, como carinho e afeto, é comum que as mães tenham diversas dúvidas a respeito do cuidado cotidiano com seu filho, necessitando assim de orientações a respeito do desenvolvimento dele, alimentação, higiene e outros fatores que favoreçam o bem-estar do binômio mãe-filho (RIQUELME CSG, et al., 2021).

Nesse sentido, o papel dos profissionais de saúde, dentre esses o enfermeiro, é orientar a mãe sobre esses cuidados, enfatizando seus benefícios e as consequências do não seguimento adequado. Uma vez que o profissional propicia atendimento integral à saúde da criança, fornece orientações, além do diagnóstico precoce de doenças prevalentes na infância, assim como o tratamento, acarretando na diminuição nas taxas de morbimortalidade infantil (SILVA GN e CARDOSO AM, 2018).

No contexto da atenção primária, a puericultura é uma prática muito eficaz no acompanhamento das crianças, principalmente porfornecer ao cuidador um espaço em que ele possa solucionar suas dúvidas e aprender a melhor forma de cuidá-las (ALVES JS, et al., 2018). Sabe-se que para o bom desempenho das mães no cuidado com o filho é importante que as orientações sejam feitas durante a consulta na unidade básica de saúde (SILVA GN e CARDOSO AM, 2018).

A atuação do enfermeiro é essencial para o desenvolvimento de uma consulta de puericultura eficaz, sendo necessário estabelecer um vínculo diante o binômio mãe/filho e que esteja preparado para orientar e suprir as demandas desse trabalho, que se evidenciam na sistematização do cuidado enfocado na vigilância do processo de crescimento e desenvolvimento, prevenção de agravos, promoção e proteção da saúde realizada desde o momento da amamentação (TAVARES MNM, et al., 2019). Apesar das orientações realizadas pelos profissionais de saúde, ainda se percebe o quanto as mães necessitam de informações sobre temáticas bem difundidas como o aleitamento materno e alimentação complementar (SOUZA THS, et al., 2021). 
Dessa forma, este artigo teve como objetivo relatar a vivência da atividade de orientações para o aleitamento materno e alimentação complementar à criança atendida na puericultura em uma unidade básica de saúde.

\section{RELATO DE EXPERIÊNCIA}

Trata-se de um relato de experiência acerca das atividades do projeto de extensão, intitulado "Orientações para o cuidado materno à criança atendida na puericultura", que foi desenvolvido em uma Unidade Básica de Saúde (UBS) de Fortaleza-Ceará.

O projeto de extensão está inserido dentro do Grupo de Pesquisa em Cuidados com a Saúde da Criança e Adolescente (GPCA) de uma Universidade do Estado do Ceará. O projeto iniciou no mês de abril de 2019, com atuação de docentes e discentes de graduação e pós-graduação do Curso de Enfermagem. Durante esse período, os integrantes participaram das atividades realizadas na UBS, por meio de oficinas interativas para as gestantes e puérperas que procuravam o serviço de saúde.

Essas oficinas foram divididas em duas temáticas, sendo elas, amamentação e alimentação complementar, as quais foram of erecidas às segundas -f eiras na unidade. A escolha do assunto foi de acordo com a demanda do serviço de puericultura, sendo importante para a promoção e proteção à saúde inf antil.

Ressalta-se que para a realização dos encontros foram feitas duas reuniões com a equipe para definir os temas mais relevantes a serem abordados. Também foi feita uma escala de divisão dos integrantes nas atividades propostas para melhor organização, contando sempre com apoio da orientadora que esteve presente em todos os encontros. Cada grupo foi responsável por elaborar a oficina de seu dia, utilizando diversos materiais, preferencialmente as metodologias ativas a fim de propiciar momentos dinâmicos e com interação entre os participantes.

Organizou-se um roteiro para a condução das oficinas. Utilizou-se o método da roda com os participantes. O método da roda é definido como uma ferramenta de cogestão, da produção em saúde de um coletivo desenvolvida com as pessoas, as quais se aplicam. Sendo também um dispositivo que potencializa a subjetividade, autonomia e protagonismo dos sujeitos, além de ampliar suas capacidades de analisar, compreender e intervir sobre si mesmo e os outros, bem como os contextos que se encontram. (PONTE HMS, et al., 2016)

A oficina foi realizada por meio de rodas de conversa com objetivo de obter uma comunicação dinâmica e produtiva, além de estimular a construção da autonomia por meio da problematização, da troca de informações e experiência entre as participantes e ministrantes da oficina. Salienta-se que foram realizadas durante o mês de agosto, conhecido mundialmente como agosto dourado, dedicado ao incentivo à amamentação.

$\mathrm{Na}$ primeira oficina sobre aleitamento materno foram abordados os seguintes temas: início da amamentação (cuidados, vantagens, número e duração de mamadas), complicações no período do aleitamento materno, sinais de alerta, ou seja, quando a mulher deve buscar um suporte profissional e banco de leite humano, enfatizando a importância dessa estratégia.

$\mathrm{Na}$ segunda oficina foram utilizados como recursos, bonecas, para apresentação da "pega" (posicionamento correto da criança e demonstração das múltiplas posições durante o aleitamento); as puérperas puderam relatar suas experiências anteriores e atuais durante a amamentação, bem como se apropriarem na prática com seus bebês sobre as técnicas de amamentação. Já as gestantes participantes, tiveram a oportunidade de aprender com as bonecas utilizadas para esse momento. Também foram utilizados slides (preferencialmente com ilustrações), cartilhas educativas usadas por um banco de leite humano, além de manuais do Ministério da Saúde (MS) que propiciaram o referencial teórico.

$\mathrm{Na}$ terceira oficina no momento da apresentação das complicações, percebeu-se as dúvidas e inquietações das participantes, bem como as opiniões divergentes entre as mesmas. Nesse momento as discentes conduziram a oficina partindo dos relatos de experiência das mães; em seguida era demonstrado, 
através de orientações na cartilha e projeções em equipamento multimídia Data Show imagens das principais complicações apontadas nos estudos (fissura, mastite, abscesso mamário, dor/trauma mamilar, ingurgitação).

Na última semana, na quarta oficina foram abordadas as políticas de incentivo ao aleitamento materno, dentre essas foi enfatizado os bancos de leite humano. Assim, foi abordada a recomendação da amamentação exclusiva até o sexto mês de vida, a doação de leite materno e as ações realizadas no banco de leite humano no intuito de promover essa iniciativa. Ao final as participantes foram conduzidas pelos discentes para conhecer a sala de apoio à amamentação implantada recentemente na UBS.

Por conseguinte, os assuntos abordados na oficina de alimentação complementar incluíram a apresentação do Guia da Alimentação Saudável para Menores de Dois Anos, dez passos da alimentação saudável para as crianças, propriedades dos alimentos e comparação do sistema digestivo adulto e infantil. Ao total foram realizados quatro encontros e a cada encontro era realizada uma atividade interativa envolvendo o assunto, dentre essas, o jogo do conhecimento que permitiu a interação e participação das mães de forma lúdica.

O jogo permitia que as participantes relatassem sobre o tema, baseado no seu conhecimento próprio, obtido por meio de suas vivências e informações concebidas no meio familiar, de amigos e das relações pessoais na região que se encontram. A partir de cada opinião e dúvida apresentada, sobre as questões levantadas do assunto em questão, as palestrantes abordaram e trouxeram o conhecimento teórico e coerente se atentando e levando em consideração as realidades que ali se apresentavam, ao longo do diálogo.

A experiência do projeto de extensão para os discentes se deu desde sua criação até sua concreta implementação. Nesta última, particularmente, os discentes que se dividiam em graduandos e graduados, puderam participar de uma experiência que trouxe inúmeros benefícios à sua formação acadêmica, desde o estudo e aprimoramento do conhecimento para ministrar as oficinas, até a observação da realidade das mães e crianças que frequentam a UBS e usuf ruem do serviço de puericultura.

As oficinas, ainda, contaram com o auxílio da equipe de Enfermagem e multiprofissional da UBS que construíram e implementaram atividades complementares a dos discentes. Esta contribuição garantiu aos alunos a experiência de vivenciar uma atuação interprofissional no cuidado à criança, prática esta que impacta positivamente na formação do enfermeiro, no sentido de que além de ensiná-lo a trabalhar em equipe, torna sua atuação mais completa e a proporciona um cuidado integral, cumprindo com um dos objetivos do Sistema Único de Saúde, a quem a UBS é vinculada.

\section{DISCUSSÃo}

As temáticas abordadas nas oficinas da extensão constituem a Política Nacional de Atenção Integral à Saúde da Criança (PNAISC) no Brasil como Eixo Estratégico II. Essa política contempla os eixos estratégicos para um cuidado integral e integrado, visando a um desenvolvimento pleno para a criança e referem à Atenção Primária à Saúde (APS) como grande fator de contribuição para reduzir a mortalidade em menores de cinco anos por causas preveníveis, como deficiências nutricionais e anemia, nas populações negra e parda (MINISTÉRIO DA SAÚDE, 2018).

No que se refere à amamentação, a maioria dos problemas relacionados à lactação pode ser prevenida com esvaziamento adequado das mamas e, quando presentes deve-se obter o manejo adequado evitandose, assim, o desmame precoce decorrente de situações dolorosas e, eventualmente, debilitantes para a nutriz (GIUGLIANI ERJ, 2014; BERNARDO FMDS, et al., 2017).

Outro estudo realizado em Cuiabá, por Gaiva MAM, et al. (2019) afirma que em casos de desmame precoce é importante que os enfermeiros enfatizem suas ações para manter o AME até os seis meses de vida e encoraje as mães a continuar amamentando após o retorno às atividades trabalhistas.

As complicações relacionadas ao AM contribuem para a falta de cumprimento das recomendaçõ es do MS, podendo ser importantes causas que interrompem a continuidade da amamentação. Desta forma os 
profissionais de saúde possuem um papel fundamental na identificação precoce, desenvolver ações compartilhadas e realizar orientações no manejo dessas dificuldades (MINISTÉRIO DA SAÚDE, 2015).

Nesse sentido, percebeu-se a necessidade de reforçar as orientações sobre essa temática para as mães durante a consulta de puericultura. Tal achado corrobora com um estudo realizado no Paraná por Stalin RRP, et al. (2019), com consultas de puericultura realizadas por enfermeiros, nesta identificou-se que foram fornecidas orientações e encorajamento ao $\mathrm{AM}$ em $89 \%$ das consultas, garantindo assim que $27 \%$ das crianças permanecessem em AME até os 6 meses de idade como preconizado pelo MS.

Acerca das condutas que podem e devem ser realizadas pelos enfermeiros diante as orientações para a amamentação estão, desmistificar mitos, instruir quanto a possíveis intercorrências e utilizar de estratégias de educação em saúde em todo ciclo gravídico-puerperal, porém para a ef etivação dessas ações e garantia de um AME é imprescindível que todos os profissionais da equipe de saúde reconheçam as vantagens do AM e estejam capacitados a orientar adequadamente os cuidadores (ANTUNES BS, et al., 2017; LUSTOSA E e LIMA RN, 2020).

Este achado ainda corrobora com um ponto considerado falho na APS, em que se relaciona com a falta de incentivo à educação permanente para o profissional de Enfermagem que lida cotidianamente com este público, tal ato motiva o enfermeiro a prestar uma consulta de puericultura muitas vezes superficial e voltada apenas aos problemas encontrados (FERREIRA AF, et al., 2019; LUCENA DBA, et al., 2018).

Nesse contexto, nota-se a importância da educação em saúde, uma vez que, constitui uma ferramenta de grande eficácia para a promoção da amamentação, além de ser reconhecida como parte das atividades do enfermeiro, principalmente no âmbito da APS e na Estratégia Saúde da Família (ESF) (MELO DF, et al., 2017; SOUZA THS, et al., 2021).

O enf ermeiro tem o papel fundamental no incentivo e na promoção da prática do aleitamento materno que devem ocorrer durante o pré-natal, pré-parto, parto, consultas de puerpério e no acompanhamento da criança no primeiro ano de vida. Portanto as ações educativas e o uso de tecnologias ilustrativas e assistidas, como ferramentas de comunicação na educação em saúde permite a inclusão social e propicia uma orientação estruturada de modo a repercutir positivamente no aleitamento materno (MODES FSSDA, et al., 2018).

Em um estudo realizado no Ceará, por Alves RMM, et al. (2019) que relata a experiência de acadêmicas de enfermagem, na realização de intervenções com mães que vivenciavam a consulta de puericultura, foi evidenciado que as alunas puderam transmitir conhecimento e informações às mães auxiliando no cuidado contínuo destas com seus filhos. Tal achado corrobora com o estudo, visto que diante a experiência vivenciada pelos integrantes, percebeu-se a importância de realizar as ações de extensão, na medida em que estas propiciam o desenvolvimento das relações interpessoais, ao mesmo tempo que permitem a troca de conhecimentos essenciais. Na qual os discentes puderam desenvolver suas habilidades em enfermagem como educadores, bem como aperf eiçoá-las na premissa de que o conhecimento fosse exposto de forma esclarecedora para todos ali presentes.

No contato dos discentes com as mães e crianças, pode-se destacar o aprimoramento de uma técnica particular à enfermagem que é o saber-fazer, descrito por Gutiérrez MGR e Morais SCRV (2017), como uma prática identitária e organizacional da enfermagem, o aprimoramento dessa habilidade pôde ser exercitado pelos estudantes ao sanarem as dúvidas das mães, ensinarem as técnicas e repassarem informações cruciais ao cuidado com a criança.

Desse modo, é perceptível que as orientações acerca do AM e AC são de suma importância para levar informação e conhecimento às gestantes e puérperas, auxiliando na melhoria da qualidade de vida do binômio mãe-filho. Por fim, a vivência na UBS durante o período do projeto de extensão reitera a necessidade e a importância de fornecer orientações acerca da amamentação e alimentação complementar, sendo fat or importante para promoção da saúde de forma ef etiva, onde as atividades de extensão são estratégias que possibilitam a relação dos discentes com a comunidade, favorecendo o aprimoramento das orientações realizadas nas atividades de educação em saúde. 


\section{REFERÊNCIAS}

1. ALVES JS, et al. Orientações sobre amamentação na atenção básica de saúde e associação com o aleitamento materno exclusivo. Ciência e Saúde Coletiva, 2018;23(4): 1077-88.

2. ALVES RMM, et al. Consulta de puericultura: o olhar sobre a prática do enfermeiro. Revista Interfaces, 2019; 7(1): $187-90$.

3. ANTUNES BS, et al. Condutas do enfermeiro na promoção da manutenção do aleitamento materno exclusivo nas consultas de puericultura. Disciplinarum Scientia, 2017;18(1):85-98.

4. BERNARDO FMDS, et al. Cuidado ao lactente para as mães em consulta de puericultura: intervenção em sala de espera. Revista de Enfermagem UFPE online, 2017;11(12):5129-38.

5. FERREIRA AF, et al. Consulta de puericultura: problemas encontrados em menores de 2 anos. Revista de Enfermagem UFPE online, 2019;13(2019): e240072.

6. GAIVA MAM, et al. Consulta de Enfermagem em puericultura na estratégia saúde da família. Revista da Sociedade Brasileira de Enfermeiros Pediatras, 2019;19(2):65-73.

7. GAUTERIO DP, et al. Puericultura em Enfermagem: perfil e principais problemas encontrados em crianças menores de um ano. Revista Brasileira de Enfermagem, 2012;65(3):508-13.

8. GIUGLIANI ERJ. Problemas comuns na lactação e seu manejo. Jornal de Pediatria, 2004;80(5): 147-54.

9. GUBERT FA, et al. Protocolo de Enfermagem para consulta de puericultura. Revista Rene, 2015;16(1):81-9.

10. GUTIRRÉZ MGR, MORAIS SCRV. Sistematização da Assistência de Enfermagem e a formação da identidade profissional. Revista Brasileira de Enfermagem, 2017;70(2):455-60.

11. LUCENA DBA, et al. Primeira semana saúde integral do recém -nascido: ações de enfermeiros da Estratégia Saúde da Família. Revista Gaúcha Enfermagem, 2018;38:1-8.

12. LUSTOSA E, LIMA RN. importância da enfermagem frente à assistência primária ao aleitamento materno exclusivo na atenção básica. Revista Brasileira Interdisciplinar de Saúde. 2020;2(2): 93-7.

13. MELLO DF, et al. Nursing care in early childhood: contributions from intersubjective recognition. Revista Brasileira de Enfermagem, 2017;70(2):446-50.

14. MINISTÉRIO DA SAÚDE. Secretaria de Atenção à Saúde. Departamento de Atenção Básica. Saúde da criança: aleitamento materno e alimentação complementar. 2015. Disponível em: https://bvsms.saude.gov.br/bvs/publicacoes/saude_crianca_aleitamento_materno_cab23.pdf. Acessado em: 08 de Novembro de 2021.

15. MINISTÉRIO DA SAÚDE. Política Nacional de Atenção Integral à Saúde da Criança: Orientações paraimplementação. 2018. Disponível em: https://portaldeboaspráticas.iff.fiocruz.br/wpcontent/uploads/2018/07/Política-Nacional-de-Atenção-Integral-à-Saúde-da-Criança-PNAISC-Versão-Eletrônica.pdf. Acessado em:20 de agosto de 2021.

16. MODES FSSDA, et al. Incentivo e Promoção do Aleitamento Materno na Consulta de Enfermagem à Criança Incentive and Promotion of Breastfeeding in the Nursing Consultation of the Child. Revista de Enfermagem Atual In Derme, 2018;86(24):1-12.

17. RIQUELME CSG, et al. Utilização de mídia social como meio de educação sobre o aleitamento materno

1. para a promoção de bem-estar de gestantes e puérperas. Brazilian Journal of Development, 2021;7(6):62349-57.

18. PONTE HMS, et al. Desafios da operacionalização do Método da Roda: experiência em Sobral (CE). Saúde Debate, 2016;40(108):34-47.

19. SILVA GN, CARDOSO AM. O papel do enfermeiro na redução da mortalidade infantil por meio do acompanhamento de puericultura na atenção básica. Revista Científica da Escola Estadual de Saúde Pública, 2018;4(1):91-9.

20. SOUZA TSHS, et al. A educação em saúde como ferramenta para promoção do aleitamento materno exclusivo. Research, Society and Development, 2021;10(6):1-11.

21. STALIN RRP, et al. Perfil das consultas de puericultura realizadas somente por enfermeiros. Revista Terra e Cultura, 2019;35(especial): 93-106.

22. TAVARES MNM, et al. Consulta de Enfermagem em Puericultura na estratégia saúde da família: revisão integrativa. Revista Nursing, 2019; 22 256): 3144-49. 